\title{
Envelope Factorization with Partial Elimination and Recombination, EF-PER, a New Linear RF Architecture
}

\author{
Antoine DIET ${ }^{1}$, Geneviève BAUDOIN ${ }^{2}$, Samson LASAULCE ${ }^{3}$ \\ ${ }^{1}$ GeePs UMR 8507 (univ. Paris Sud, CNRS, CentraleSupélec, UPMC), rue Joliot Curie 91192 Gif S/ Yvette, France \\ ${ }^{2}$ Université Paris-Est, ESYCOM (EA2552), ESIEE Paris, UPEMLV, CNAM. F-93162, Noisy-le-Grand, France \\ ${ }^{3}$ L2S UMR 8506 (CNRS, univ. Paris Sud, CentraleSupélec), rue Joliot Curie 91192 Gif S/ Yvette, France
}

antoine.diet@geeps.centralesupelec.fr, genevieve.baudoin@esiee.fr, samson.lasaulce@1ss.centralesupelec.fr

\begin{abstract}
In this paper, a new architecture for efficient linear radio frequency transmitters is proposed; it includes envelope-tracking (ET) and envelope-elimination-andrestoration (EER) architectures as special instances. The proposed technique is referred to as Envelope Factorization with Partial Elimination and Recombination (EFPER). It relies on a decomposition of the RF signal before power amplification as a product of two signals, one of them being the envelope signal elevated to an exponent " $\alpha$ ". Compared to ET or EER architectures, the parameter " $\alpha$ " constitutes a new degree of freedom. This allows one to realize good tradeoffs between different performance criteria such as spectrum use, power efficiency, and transmitter linearity. An intuitive aggregate cost function is introduced to capture the desired tradeoff and turns out to be maximized in $\alpha=0.5$. The full relevance of EF-PER is sustained both by analytical results and realistic simulations performed for OFDM signals. The EF-PER architecture (with $\alpha=0.5$ ) has been simulated under Agilent$A D S$ with a non-linear transistor model from Avago (E-PHEMT) and compared with ET and EER.
\end{abstract}

\section{Keywords}

EER, ET, linearization techniques, power efficiency, PAPR, RF architecture, RF linear transmitter

\section{Introduction}

Many modern communication and broadcasting systems, such as the LTE (long term evolution), rely on OFDM (orthogonal frequency division multiplexing) technology, mainly for its good spectral efficiency. Unfortunately, the peak to average power ratio (PAPR) of OFDM signals is high (typically of the order of $10 \mathrm{~dB}$ ), which makes the design of transmitters with good linearity and efficiency difficult. Indeed, typical power amplifiers (PA) achieve maximum power efficiency when the maximum output voltage swing is reached and in that case, input levels are close to the saturation where the PA is non-linear. To improve linearity it is possible to impose the power amplifier (PA) to operate with a backoff from its saturation point but this is highly detrimental to the power efficiency. The higher the PAPR, the higher is the necessary backoff and the lower the power efficiency. Several approaches [1], [2], [3], [4] were investigated to improve the tradeoff between linearity and efficiency by modifying the input signal (crest factor reduction, predistortion), the PA design [1] (Doherty, Switch-mode PA, DPA/RFDAC) or the transmitter architecture and the PA design at the same time (LINC/CALLUM, EER, ET).

It is possible to decrease the PAPR of OFDM signals by using techniques such as clipping or more sophisticated crest factor reduction methods. It allows one to decrease the backoff and therefore to increase the efficiency but this is most of the time detrimental to the signal quality.

Digital PreDistortion (DPD) [5] consists in distorting the input signal by a baseband predistorter whose characteristics are the inverse of those of the PA. It is currently one of the most effective linearization methods and it is widely used for base station transmitters in cellular communications and broadcasting. In some cases, it can improve the efficiency by decreasing the necessary backoff. However, it cannot provide this high efficiency over a wide range of variation of the signal mean power. Additionally, the computational load of DPD is important and it may increase the power consumption of the baseband part of the transmitter. This is negligible for high power transmitters of base stations but not for mobile handsets. Consequently, other efficiency improvement techniques, mentioned below, can not only be an alternate solution but fruitfully combined with DPD [6].

Doherty power amplifiers [7] are based on an active load modulation principle that allows one to improve the transmitter efficiency for high PAPR signals. Doherty PAs are currently associated with DPD in base station transmitters. Their main shortcomings are bad linearity, size of the structure (two or more PAs are used with impedance converters) and limitation in terms of bandwidth and frequency flexibility. For those reasons, this solution is not used in mobile handsets.

Switch-mode PAs [1], [2], [8-10] are based on operating the transistors in saturation and avoiding overlap in 
time between voltage and current, in order to minimize power dissipation. They are a potential solution for high efficiency amplification, but the transistor technology limitation in frequency impacts the performances. Besides, the architecture using this type of PA has to be modified to take into account the envelope amplitude variation. One approach is to encode the amplitude variation, with Pulse Width Modulation (PWM) or $\Sigma \Delta$ coding [4], [11], in order to suppress the dynamic. In these cases, a filtering of the RF signal is mandatory, at the PA output, to meet the spectrum mask requirements of the standards. This filtering reduces the system efficiency.

Digital PA and RF-DAC [4], [9], [12] can be used at low RF power of emission (Bluetooth for example) but they present the same kind of technological limitations and filtering needs as switch-mode PAs.

Other solutions are architectures based on a decomposition of the RF input signal $x(t)=A(t) \cos (\omega t+\varphi(t))$ into a sum or a product of two components), which are amplified separately in an efficient way and then recombined. This decomposition modifies the signal characteristics (frequency spectrum, statistical properties...) and consequently impacts the PAs design. We can cite LINC (LInear amplification with Non-linear Component), EER and ET architecture.

LINC [13-15] is based on decomposing $x(t)$ into a sum of two constant amplitude component signals that can be amplified by 2 RF PAs in an efficient manner since their PAPRs are low. This is obtained at the expense of the signals bandwidths which are widened by this decomposition. The main limitations or challenges are the necessity to design identical amplifiers paths for the two signals and the difficulty to design a non-isolating lossless power combiner (since for isolating power combiners the losses can reach $3 \mathrm{~dB}$, which limits the maximum efficiency to $50 \%$ ). For these reasons, this method is not widely used currently.

EER [1], [2], [4], [16-18] relies on a factorization of $x(t)$ into a product of a constant amplitude bandpass signal $x_{2}^{\prime}(t)=\cos (\omega t+\varphi(t))$ and a baseband envelope signal $x^{\prime}{ }_{1}(t)=A(t)$. The constant amplitude bandpass signal $x_{2}^{\prime}(t)$ can be amplified with an RF PA which operates in a high efficiency class. The baseband signal, $x_{1}^{\prime}(t)$ is amplified by a highly efficient baseband PA (e.g., a switching mode class) and is used to modulate in amplitude the supply voltage of the RF PA, in order to recombine the signal components $x_{1}^{\prime}(t)$ and $x_{2}{ }_{2}(t)$. The overall efficiency is mainly the product of the two PAs efficiencies. In such recombination, the time mismatch is critical [4], [19], [20]. The bandwidths of the signals $x_{2}^{\prime}(t)$ and $x_{1}^{\prime}(t)$ are wider than that of the original signal $x(t)$, highly challenging the design of the baseband PA. Another difficulty arises when lowest values of the envelope occur on the supply voltage while the PA has a constant input RF power. This argues for a variation of the input RF signal in function of the envelope.

ET [6], [10], [17], [21-26] architecture is a kind of dynamic biasing techniques. It is built on the association of two signals: the baseband envelope signal $x{ }_{1}(t)=A(t)$ (like EER) and a bandpass signal $x{ }_{2}(t)=$ $A(t) \cos (\omega t+\varphi(t))=x(t)$; the envelope signal is used to modify the supply voltage of the RF PA in order to track the envelope signal and optimize the power efficiency. The gain and efficiency of the ET architecture depends on the supply voltage and RF signal input power in a complex way, inherent to the used PA. Therefore, a mapping has to be calculated to determine an optimal relation between the envelope signal and the supply voltage for a given criterion: maximum efficiency or constant gain for example. The mapped envelope signal is amplified by a baseband highly efficient PA which design's considerations are almost similar for EER and ET cases.

The new architecture EF-PER proposed in this paper, is built on a factorization into two signals, as in the case of EER and ET, but the baseband modified envelope signal is taken to be $x_{1}(t)=A(t)^{\alpha}$, while the bandpass signal is taken to be equal to $x_{2}(t)=A(t)^{\beta} \cos (\omega t+\varphi(t))$. Clearly, the cases $(\alpha, \beta)=(1,0)$ and $(\alpha, \beta)=(1,1)$ respectively correspond to EER and ET. These new degrees of freedom $(\alpha, \beta)$ can therefore be used to share the constraints between the RF and baseband paths in terms of input signal PAPR and bandwidths. We show, in the following parts, that EF-PER can be used for signals with higher bandwidths than in EER and ET cases. Also, the EF-PER is less impacted than EER by a time mismatch between the two signals paths.

The paper is organized in four sections including the introduction. Section 2 describes EF-PER and provides several analytical results on the signals under consideration. Section 3 provides electrical simulations with a nonlinear transistor model. This allows us to assess the performance of the proposed technique, mainly in terms of power efficiency versus output power. Section 4 concludes the paper.

\section{EF-PER Linearization Technique}

\subsection{Principle of the EF-PER}

In this section, we present the EF-PER architecture, we analyze the statistical properties of the signals components and compare them with those of EER and ET.

$$
\begin{gathered}
x(t)=g\left(x_{1}(t, \alpha)\right) x_{2}(t, \beta), \\
x_{1}(t, \alpha)=A(t)^{\alpha} \text { and } x_{2}(t, \beta)=A(t)^{\beta} \cos (\omega t+\varphi(t))
\end{gathered}
$$

The key idea of EF-PER is to build an amplified modulated signal, $x(t)=A(t) \cos (\omega t+\varphi(t))$, from the combination of two elementary signals $x_{1}(t, \alpha)$ and $x_{2}(t, \beta)$ that are amplified separately: a baseband modified envelope signal, $x_{1}(t, \alpha)=A(t)^{\alpha}$, and a bandpass signal, $x_{2}(t, \beta)=$ $A(t)^{\beta} \cos (\omega t+\varphi(t))$, see (1a). A mapping function $g($.$) can$ be applied to $x_{1}(t)$ to take into account the PA gain variation with supply voltage. 


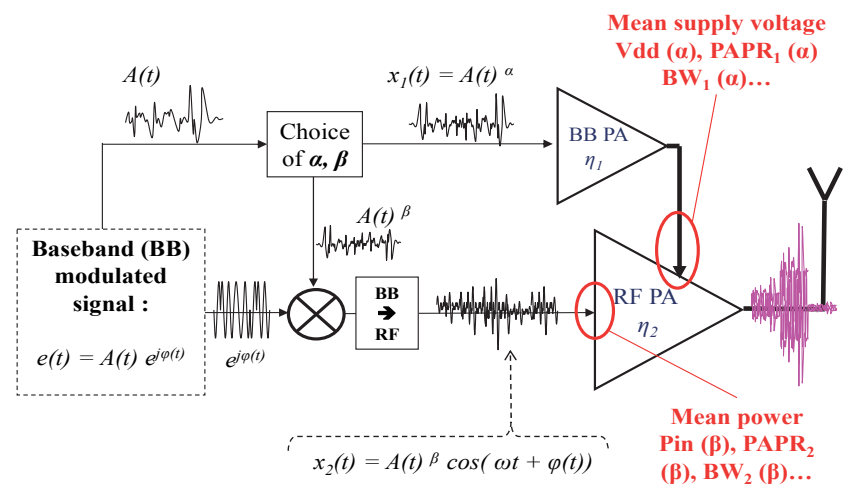

Fig. 1. The EF-PER technique. The first signal component controls the PA supply voltage. The second one is the input of the RF PA.

Fig. 1 describes the EF-PER method, where $x_{1}(t, \alpha)$ controls the PA supply voltage, and $x_{2}(t, \beta)$ is the input of the RF PA. The choice made in this paper is to assume $\beta=(1-\alpha)$ and a linear gain variation for $g($.$) , and assess$ the corresponding performance thoroughly. Although, by doing this, we move from two degrees of freedom to only one, we will see that this choice is sufficient to reach the set objectives namely, obtaining a large range of possible tradeoffs between EER and ET. This corresponds to:

$$
x_{1}(t, \alpha) x_{2}(t, 1-\alpha)=A(t)^{\alpha} A(t)^{(1-\alpha)} \cos (\omega t+\varphi(t))=x(t) .
$$

As $\alpha$ is a single parameter, we write $x_{1}(t, \alpha)=x_{1}(t)$ and $x_{2}(t, 1-\alpha)=x_{2}(t)$.

\subsection{Analysis of Signals Characteristics}

Performances of the structure depend on the characteristics of the input signal $x(t)$ and, more precisely, of its components $x_{1}(t)$ and $x_{2}(t)$. In this paper, we consider OFDM signals because of their wide use in modern systems. In practice, there are at least four important criteria for the characterization of signal components:

- PAPR impacts directly the efficiency since the higher the PAPR, the higher the backoff.

- Cubic Metric [27] and, in our case, Raw Cubic Metric (RCM), is a good criterion for quantifying the power de-rating of the PA when driven by (OFDM) modulated signals, as suggested in 3GPP framework.

- Bandwidth is a critic characteristic, especially for the envelope path with a switch-mode PA. In our simulations, we have defined the bandwidth as the frequency range containing $99 \%$ of the total power.

- Normalized variance (NVAR) is the variance divided by the square mean. It quantifies the dynamic variation (statistical) of the PA operating point. It is easier to optimize a PA to a signal whose variance is limited, around the nominal value. The variance directly increases the difficulty in maintaining PA gain constant, high output power and high efficiency. Some signals can have the same PAPR with different NVAR, impacting differently the PA gain, output power and average efficiency. This feature is complementary to PAPR and RCM because it highlights the electrical behavior variation of the circuits (mainly PAs) in the transmitter.

We have simulated PAPR, RCM and bandwidth for the two signals of EF-PER, in function of $\alpha$. Also, we have derived analytically the statistical properties of $x_{1}(t)$ and $x_{2}(t)$ signals in the case where the envelope signal $A(t)=$ $|x(t)|$ is Rayleigh distributed (that is a good model for the envelope of OFDM signals with several tens of carriers) and given the closed-form expressions of their mean, variance and normalized variance. The knowledge of the probability density function is necessary to calculate the average efficiency $(\eta)$ in function of the input power $P_{\text {in }}$.

All the simulations under MATLAB have been performed for 256 OFDM symbols with 1024 sub-carriers and a 64-QAM modulation, at an oversampling rate of 16 which corresponds to more than 4 billion samples for $x(t)$. The shaping filter is a raised cosine (roll-off $=0.5$ ).

Equations (2a) and (2b) define the PAPR and RCM of $x_{1}(t)$ and $x_{2}(t)$. For simplicity in the analysis, we have expressed $P A P R_{1}$ and $P A P R_{2}$ in baseband even when $x_{2}(t)$ is a bandpass RF signal. Fig. 2 depicts $P A P R_{1}, R C M_{1}, P A P R_{2}$ and $R C M_{2}$ as a function of $\alpha$. In this technique, the PAPR of the original signal $x(t)$ is distributed between the two signals $x_{1}(t)$ and $x_{2}(t)$. This is why we have called this technique "partial elimination".

$$
\begin{aligned}
P A P R_{1}= & \frac{\max \left|x_{1}(t)\right|^{2}}{E\left(\left|x_{1}(t)\right|^{2}\right)}=\frac{\max \left|A(t)^{\alpha}\right|^{2}}{E\left(\left|A(t)^{\alpha}\right|^{2}\right)}, \\
P A P R_{2}^{R F}= & \frac{\max \left|x_{2}(t)\right|^{2}}{E\left(\left|x_{2}(t)\right|^{2}\right)}=\frac{\max \left|A(t)^{(1-\alpha)} \cos (\omega t+\varphi(t))\right|^{2}}{E\left(\left|A(t)^{(1-\alpha)}\right|^{2}\left[\cos ^{2}(\omega t+\varphi(t))\right]\right)} \\
= & 2 \frac{\max \left|A(t)^{(1-\alpha)}\right|^{2}}{E\left(\left|A(t)^{(1-\alpha)}\right|^{2}\right)}=2 P A P R_{2}, \\
& R C M\left(x_{i}(t)\right)=R M S\left(\frac{x_{i}(t)}{R M S\left(x_{i}(t)\right)}\right)^{3} .
\end{aligned}
$$

In Fig. 2, the worst cases for $P A P R_{1}$ and $P A P R_{2}$ correspond to the ET case, where both signals reach $12 \mathrm{~dB}$. $R C M_{1}$ and $R C M_{2}$ present almost the same behavior as $P A P R_{1}$ and $P A P R_{2}$ in function of $\alpha$. For the EER case, only $P A P R_{1}$ reaches this maximum value of $12 \mathrm{~dB}\left(P A P R_{2}=\right.$ $0 \mathrm{~dB})$. Reducing the original PAPR or RCM of $x(t)$ by sharing it out between $x_{1}(t)$ and $x_{2}(t)$ can be fruitful for improving PA performance (e.g. for $\alpha=0.5$, both PAPR are equal to $6 \mathrm{~dB}$ ). 


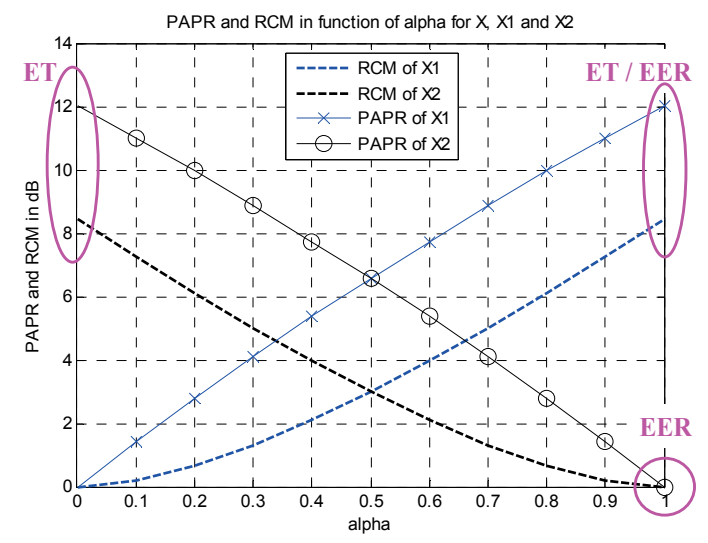

Fig. 2. PAPR and RCM of $x_{1}$ and $x_{2}$ in function of $\alpha$. Corresponding values for EER and ET cases are marked.

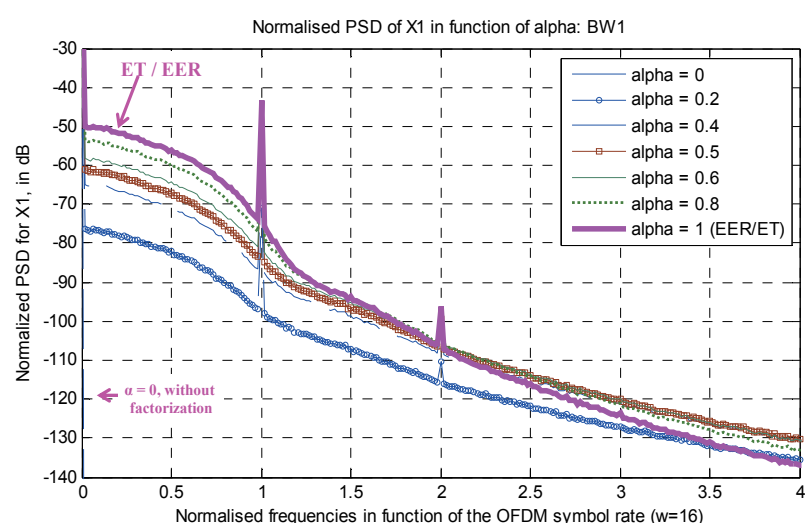

Normalised PSD of $\mathrm{X} 2$ in function of alpha: BW2

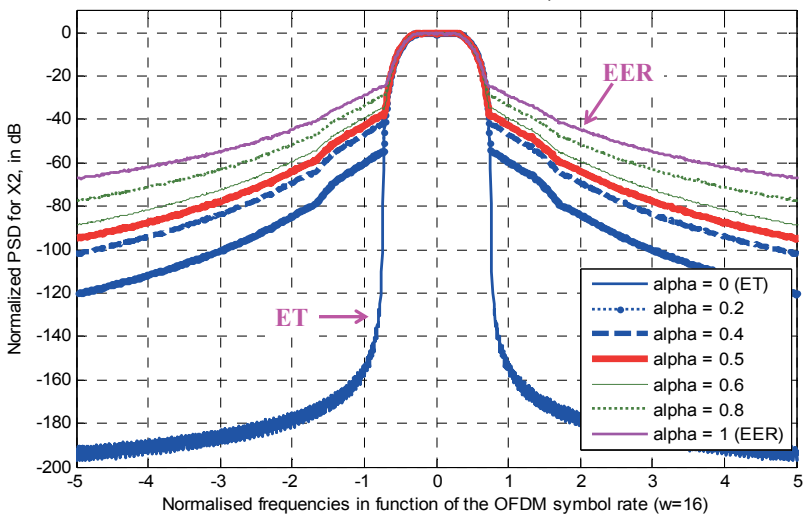

Fig. 3. Spectrum of $x_{1}(t)$ (top) and $x_{2}(t)$ (bottom). The $x_{1}(t)$ signal bandwidth is widening with $\alpha$. Increasing $\alpha$ means reducing the envelope exponent in $x_{2}$ expression. The $x_{2}$ "phase signal" is for $\alpha=1$, EER case.

Implementing an elimination (even partial) and recombination method implies a bandwidth widening effect due to the use of non-linear operators, such as calculation of the magnitude $A(t)$ or its elevation to a power $\alpha$. Fig. 3 represents the Power Spectral Densities (PSD) of $x_{1}(t)$ (baseband signal) and $x_{2}(t)$ (bandpass signal). In Fig. 4, we have plotted (thanks to a cumulative sum in the simulation) the bandwidths corresponding to $99 \%$ of the power of the signals $x_{1}(t)$ and $x_{2}(t): B W 1(\alpha)$ and $B W 2(\alpha)$ respectively.

The signal $x_{1}(t)$ is always positive; consequently its PSD comprises a continuous PSD and a sum of Dirac

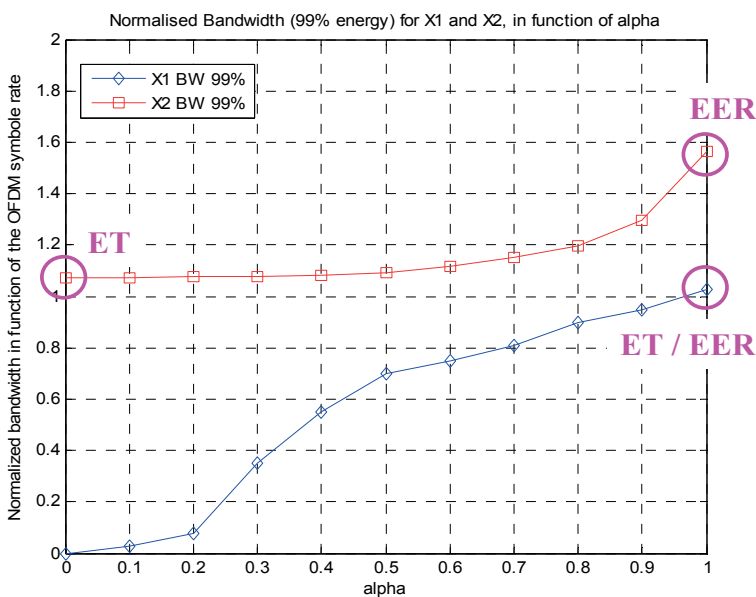

Fig. 4. BW1 and BW2 in function of $\alpha$. ET and EER values are marked. Both bandwidths are increasing with $\alpha$.

masses which are located according to the multiples of the symbol rate. The PSD of $x_{1}(t)$ is widening while $\alpha$ is increasing from 0 to 1 . Choosing $\alpha=0$ implies that $x_{1}(t)$ is a constant value, and its PSD is a Dirac mass centered at the origin, and the case $\alpha=1$ corresponds to the PSD of the envelope signal $A(t)$. EER and ET corresponds to worst cases for the bandwidth of $x_{1}(t)$, see Fig. 3 .

The bandwidth of $x_{2}(t)$ increases with $\alpha$. For $\alpha=1$, $x_{2}(t)$ corresponds to the phase signal of the EER case, and presents the maximum bandwidth. ET case corresponds to the minimum bandwidth $(\alpha=0)$ and EER is the worst case.

EF-PER values for $B W 1$ and $B W 2$ are increasing with $\alpha$. We notice that ET leads to: $B W 1(1)$ and $B W 2(0)$, while EER represents an extreme case: $B W 1(1)$ and $B W 2(1)$. As said in the introduction, the EF-PER can especially reduce the bandwidth of the envelope signal path $x_{1}(t)$, compared to EER and ET cases.

When varying $\alpha$, the signals $x_{1}(t)$ and $x_{2}(t)$ present different laws of probability. Statistical properties are very important for the PA because they determine the variation around the device operating point. We focus on the signals normalized variance (NVAR) for the component signals $x_{1}(t)$ and $x_{2}(t)$, respectively $\mathrm{NVAR}_{\mathrm{X} 1}$ and $\mathrm{NVAR}_{\mathrm{X} 2}$. We can express the density probability function of $x_{1}(t)$ and $x_{2}(t)$ under the assumption that an OFDM signal can be considered as a centered complex Gaussian signal $I+j Q$ where $I$ and $Q$ are independent and with a variance $\sigma^{2}$. Analytically, $A(t)$ is defined by a Rayleigh distribution and equation (3) gives the probability density for $A(t)^{\alpha}$ which is calculated in the appendix.

$$
f_{x_{1}=A^{\alpha}}(u)=\frac{1}{\alpha \cdot \sigma^{2}} u^{\frac{2-\alpha}{\alpha}} \exp \left(-\frac{u^{\frac{2}{\alpha}}}{2 \sigma^{2}}\right) .
$$

Equation (4) provides the expression of the statistical mean and variance of the factorization signal $(\Gamma$ is the "gamma" function). The mean and variance of $x_{1}(t)$ are highly dependent on $\alpha$ and $\sigma$. Also, the NVAR values, for $\alpha=0.9$ and $\alpha=0.4$ correspond to a division by 4 . 


$$
\begin{aligned}
m_{x_{1}=x^{\alpha}} & =\left(2 \cdot \sigma^{2}\right)^{\alpha / 2} \cdot \Gamma\left(1+\frac{\alpha}{2}\right)=m_{x_{1}}, \\
V A R_{x_{1}=x^{\alpha}} & =m_{x_{1}}^{2}+\left(\alpha \cdot 2 \cdot \sigma^{2}\right) \cdot K, \\
K & =\left(2 \cdot \sigma^{2}\right)^{(\alpha-1)} \Gamma(\alpha)-\left(2 \cdot \sigma^{2}\right)^{\left(\frac{\alpha}{2}-1\right)} \Gamma\left(\frac{\alpha}{2}\right) m_{x_{1}}, \\
N V A R_{x_{1}=x^{\alpha}} & =\frac{V A R_{x_{1}=x^{\alpha}}}{m_{x_{1}}^{2}}=1+\frac{\left(\alpha \cdot 2 \cdot \sigma^{2}\right) \cdot K}{m_{x_{1}}^{2}} \\
& =1+\alpha\left[\Gamma(\alpha)-\Gamma\left(\frac{\alpha}{2}\right) \Gamma\left(1+\frac{\alpha}{2}\right)\right]\left[\Gamma\left(1+\frac{\alpha}{2}\right)\right]^{-2} .
\end{aligned}
$$

In EER and ET, the variance (and also PAPR and $R C M$ ) of the envelope signal $x_{1}(t)$ corresponds to worst cases. For the signal $x_{2}(t)$, ET is the worst case while EER is the best case. The statistical analysis therefore shows one advantage of EF-PER compared to ET and EER for $x_{1}(t)$ in reducing the normalized variance.

To conclude this sub-section, we have seen that EER has the advantage of reducing the PAPR and RCM of $x_{2}(t)$ but at the price of enlarged bandwidths for the signals. ET is the solution which presents the minimum bandwidth for $x_{2}(t)$ but implies a high PAPR on the two signals $x_{1}(t)$ and $x_{2}(t)$. Both EER and ET have high PAPR for the baseband signal $x_{1}(t)$. EF-PER can be seen as an intermediate solution which allows to trade-off the aforementioned advantages. Especially, it allows reducing the PAPR/RCM and the bandwidth of $x_{1}(t)$ compared to ET and EER. It also reduces the PAPR/RCM of $x_{2}(t)$ compared to ET.

\subsection{Cost Function for the Choice of $\alpha$}

EF-PER can fruitfully share the constraints (PAPR, RCM, bandwidth, NVAR) on the signals $x_{1}(t)$ and $x_{2}(t)$ and is parameterized by $\alpha$. To find an optimum value for $\alpha$, we propose a cost function, $F$, constructed as the sum of two elementary cost functions, $F_{1}$ and $F_{2}$, respectively corresponding to $x_{1}(t)$ and $x_{2}(t)$. Each elementary cost function is the product of PAPR, RCM, bandwidth and normalized variance of the signals, as seen in (5). Different cost functions can be defined, but we tried here to set one function $F$ that enables a quantitative comparison of the different architectures, taking into account all the above mentioned characteristics of the signals impacting on the PA design. Note that all quantities which intervene in the expression of $F_{1}$ and $F_{2}$ have to be minimized: $B W, P A P R, R C M$ and $N V A R$, which justifies the multiplicative structure of the proposed performance criterion.

$$
F=F_{1}+F_{2} \text { with } F_{i}=B W_{i} \cdot P A P R_{i} \cdot R C M_{X_{i}} \cdot N V A R_{X_{i}} \cdot
$$

$F_{1}(\alpha), F_{2}(\alpha)$ and $F(\alpha)$, respectively $F_{1}, F_{2}$ and $F$, are plotted in Fig. 5. The value of $F$ for ET is not represented because it reaches 18.2 due to the high PAPR and RCM values of the two signals $x_{1}(t)$ and $x_{2}(t)$. Figure 5 reveals

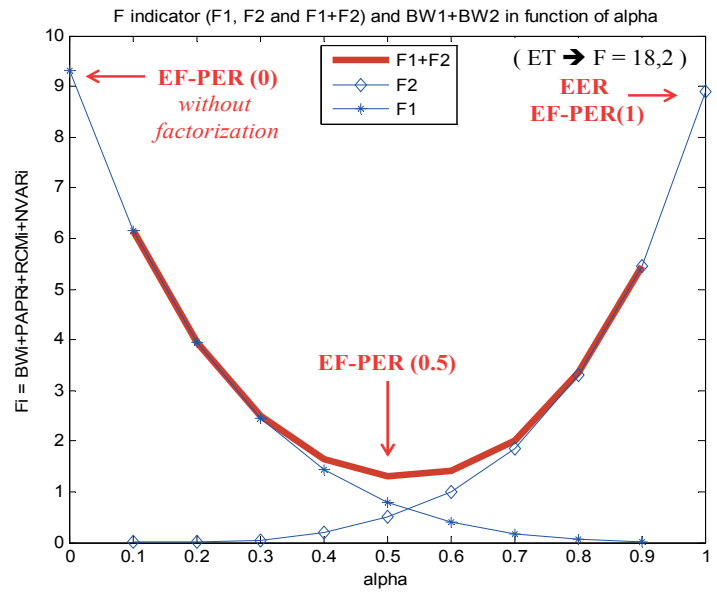

Fig. 5. Cost functions values, in function of $\alpha$, see (5).

an optimum value for $\alpha=0.5$. Nevertheless, this cost function averages different criteria and $\alpha=0.5$ is not optimal for each individual parameter, e.g. the bandwidth of $x_{2}(t)$ is increased compared to ET.

\subsection{Some Implementation Considerations}

The complexity of the implementation of the method is similar to that of EER and ET. The elevation to the power " $\alpha$ " of the envelope signal can be done by the use of Look Up Table (LUT). The consumption and complexity involved by using this LUT can be compared to that of the mapping function in the ET architecture.

Another important point to consider is the sensibility to a time mismatch between the two signals $x_{1}(t)$ and $x_{2}(t)$ at the recombination. As it is highly impacting the spectrum in practice, especially for the EER architecture [2], [4], [17-20] we have plotted in Fig. 6 the PSD, normalized in function of its maximum value, of the recombined signal, for an arbitrary time mismatch of $3 T_{\mathrm{s}} / 16$. As the spectrum is more widened if $\alpha$ increases, results show that EFPER, for $\alpha<1$, is less impacted than EER by the time mismatch at the recombination.

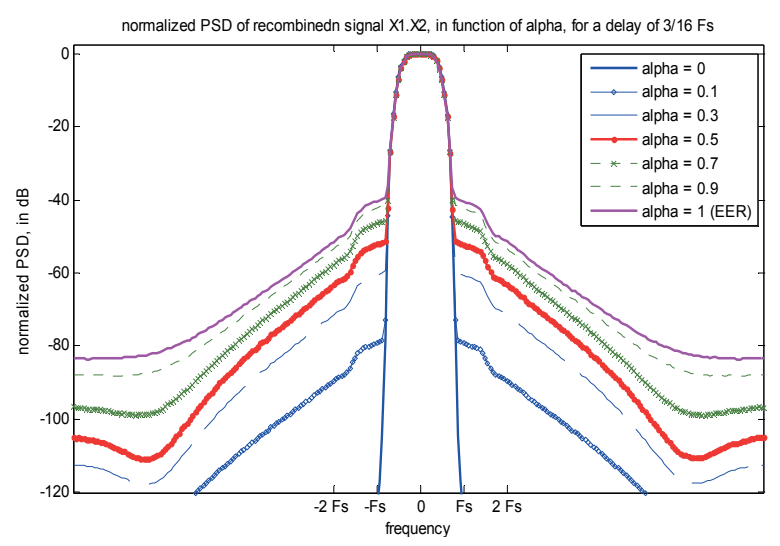

Fig. 6. Normalized PSD of the recombined signal, for different value of $\alpha$, with a time mismatch of $3 / 16$ of a symbol period (1/Fs). 


\section{Performances Evaluation with an Electrical Non-Linear PA Model}
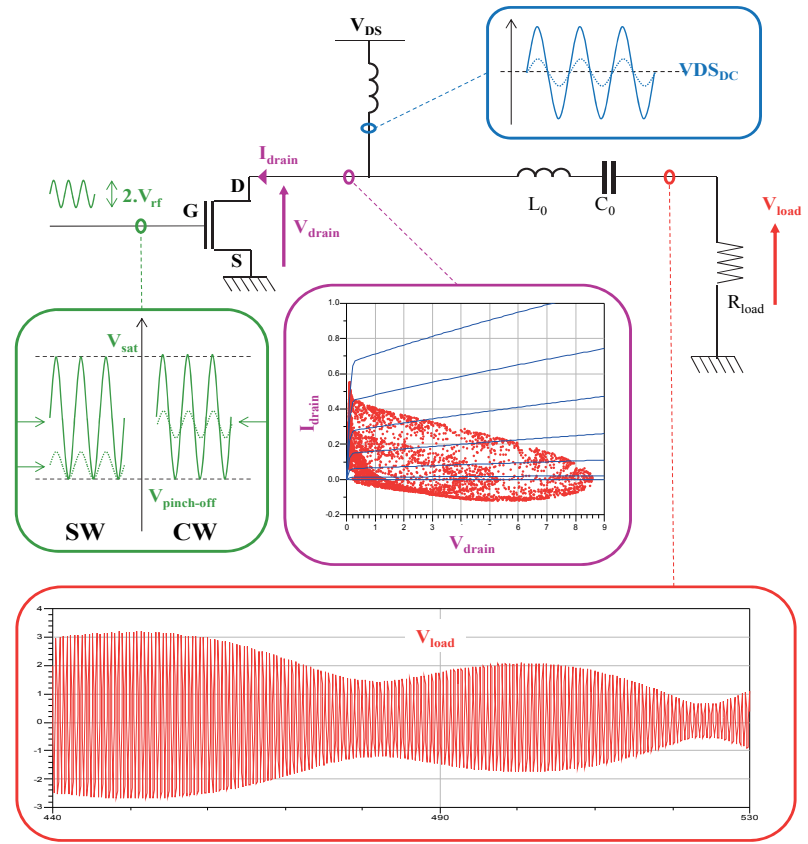

Fig. 7. Simulation topology of the $2 \mathrm{GHz}$ RF PA. The transistor is biased in SW or $\mathrm{CW}$ mode and its drain voltage can be modulated.

We have simulated the EF-PER architecture (with $\alpha=0.5$ ) under Agilent-ADS with a realistic electrical nonlinear transistor model and compared this architecture with ET and EER. As seen in Fig. 1, there are two PAs to consider: the RF PA (bandpass) and the baseband PA. The latter has to generate the supply voltage of the RF PA, while amplifying the envelope (or partial envelope) information. The signals recombination is achieved by supply modulating the RF PA. The electrical simulation has been performed at the RF PA level and an ideal envelope amplifier has been assumed. One of the greatest interests of EF-PER is to facilitate the design of the envelope amplifier since it reduces the PAPR/RCM and bandwidth of $x_{1}(t)$. Therefore, we focus this electrical simulation on the RF PA.

An RF PA has been simulated under Agilent-ADS with a non-linear AVAGO transistor model (E-PHEMT). This PA was tuned at $2 \mathrm{GHz}$. While modifying the RF driving signal and the supply-modulated voltage, the size of the transistor is fixed, whatever the architecture. Fig. 7 shows the electrical simulation circuit topology. We consider two cases for classes of operation: "continuous wave class" (CW, such as A, AB classes) for which the small-signal voltage $v_{r f}$ varies around a bias DC voltage $V_{0}$ and "highly saturated class B and switched classes" (SW) for which the voltage $v_{r f}$ varies from $V_{\text {pinch-off }}$ to the maximum of voltage excursion. The modulation of the driving signal and supply voltage are qualitatively represented in Fig. 7 (for sinusoidal voltage at different levels), as seen on the representation of $V_{\text {load }}$. In that figure, the load-line ( $I_{\text {drain }}$
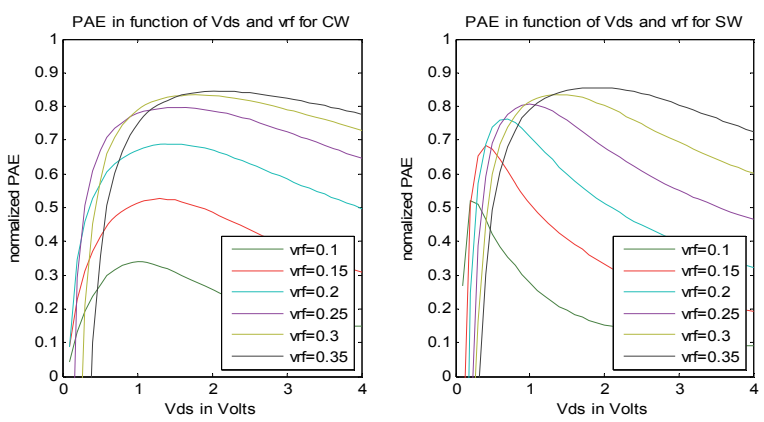

Fig. 8. PAE for $\mathrm{CW}$ mode (left) and $\mathrm{SW}$ mode (right) under Agilent-ADS ( with Avago model) in function of $v_{\mathrm{rf}}$ and $V_{\text {Ds. }}$.

versus $V_{\text {drain }}$ ) illustrates the transistor dynamic driving. Electrical performances $P_{\mathrm{RF}}$ (output power) and $\eta$ (efficiency) are evaluated with classical sinusoidal formulae in that paper. In a first step, the supply voltage $V_{\mathrm{DS}}$ and the driving input voltage, $V_{\mathrm{GS}}=V_{0}+v_{\mathrm{rf}}$, are varied for $\mathrm{SW}$ and $\mathrm{CW}$ classes. Power Added Efficiency $(P A E)$ is reported in Fig. 8 for different values of $v_{\mathrm{rf}}$ and $V_{\mathrm{DS}}$.

In $\mathrm{CW}$ mode, the generation of dissipated power is highly linked to the value of $v_{\mathrm{rf}}$ because the transistor is biased. That is why the maximum $P A E$ is achieved for high values of $v_{\mathrm{rf}}$, which means for a transistor hardly driven at its input (saturation).

In $\mathrm{SW}$ mode, the transistor is driven by setting the value of $V_{0}$ equal to $v_{\mathrm{rf}}$, implying the lowest value of $V_{\mathrm{GS}}$ to be " 0 " (pinch off voltage for this E_PHEMT). The output power is maximized at the saturation (as for CW). Efficiency is high for high values of $v_{\mathrm{rf}}$.

An optimization of the dynamical driving operation of the transistor is to control the variation of $V_{\mathrm{DS}}$ and $v_{\mathrm{rf}}$ in order to produce the desired output power while maximizing the efficiency.

EER, ET and EF-PER are different manners of varying $V_{\mathrm{DS}}$ and $v_{\mathrm{rf}}$, and, consequently, represent different possibilities in driving the transistor. The emitted signal is supposed to be high PAPR OFDM. Consequently, supply voltage and input signal $v_{\mathrm{rf}}$ are varied with power distributions discussed in the previous part. Also, different mean/ average power levels are possible as potential trajectories in the $\left(V_{\mathrm{DS}}, v_{\mathrm{rf}}\right)$ plane. If we fix the horizontal axis for $V_{\mathrm{DS}}$ and the vertical axis for $v_{\mathrm{rf}}$, as in Fig. 9, we can describe the trajectory of each method ET, EER and EF-PER. In this figure, the power distribution inside these ellipses presents a high PAPR and the mean value is spotted. EER ellipse is horizontal. EF-PER, due to the modification of the signals presented in the previous section, is described by an ellipse differently orientated and less extended than ET and EER. Thus, it can be adapted to the electrical characteristics of the transistor in the $\left(V_{\mathrm{DS}}, v_{\mathrm{rf}}\right)$ plane. Consequently, the tradeoff between efficiency and output power can be fulfilled more easily.

In order to confirm the observations, the PA is driven with the modulated OFDM signal in the EER, ET and EFPER $(\alpha=0.5)$ cases, at different power levels by setting $v_{\mathrm{rf}}$ 


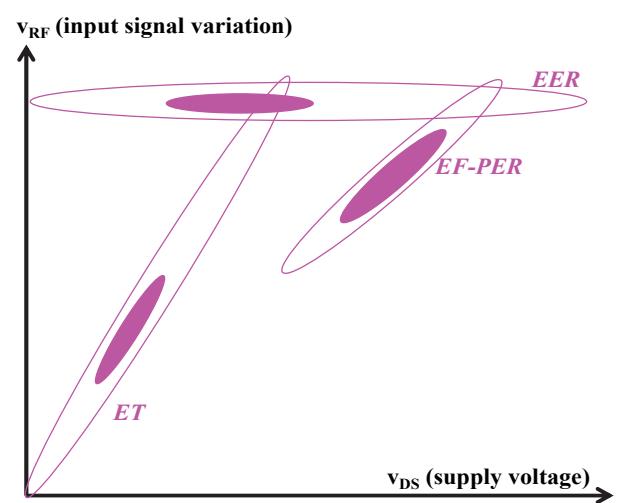

Fig. 9. Possible trajectories for EER, ET and EF-PER in $\left(V_{\mathrm{DS}}\right.$, $\left.v_{\mathrm{rf}}\right)$ plane.

and $V_{\mathrm{DS}}$ maximal values, and for both $\mathrm{SW}$ and $\mathrm{CW}$ classes of operation. The transistor size, tuning circuit and load remain unchanged for all of these simulations. Fig. 10 reports the results of these simulations in terms of power efficiency versus output power. Three areas are delimited in Fig. 10: A, B and C.

Area $\mathrm{C}$ is the highest efficiency amplifications, at low power level, including EER and EF-PER examples. It is important to notice that we can make an efficient power control without focusing exclusively on maximum power generation. In fact, the power variation in wireless networks is often at medium and low level due to the power dynamical control. Area B presents a medium power and efficient amplification, in the range of $85 \div 88 \%$, where the EER, ET and EF-PER examples are present. Area A delimits the highest power obtained. In that area, only EFPER examples reaches more than $80 \%$ efficiency. Finally, the examples of area $\mathrm{A}, \mathrm{B}$ and $\mathrm{C}$ confirm the qualitative analysis in which the output power at high efficiency is more easily reachable with EF-PER. Another interpretation is possible: for a given output power, the size of the transistor used in EF-PER will be potentially lower than for EER and ET, resulting in lower transistor output parasitic effects and higher transition frequency $\left(F_{\mathrm{t}}\right)$.

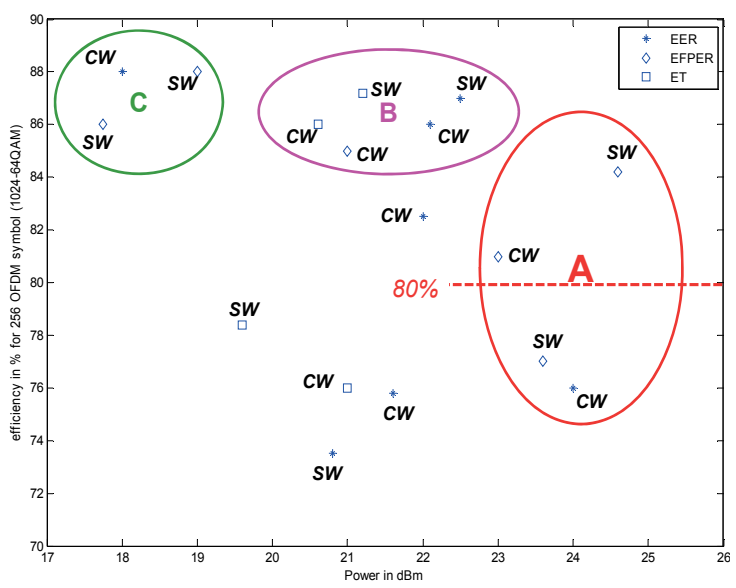

Fig. 10. PA efficiency versus $P A$ output power, for EER, ET, EF-PER case, and for the same transistor in SW or CW mode (dynamical variation of $V_{\mathrm{DS}}$ and $v_{\mathrm{rf}}$ with the simulated OFDM signal).
To conclude this part, simulations based on a non-linear transistor model, and for a high PAPR OFDM signal, shows that the performances of the RF PA can be optimized by choosing the EF-PER method. Also EF-PER allows reducing transistor size since it delivers more power for a given efficiency. This new architecture enables the definition of different PA operating point $\left(V_{\mathrm{DS}} / v_{\mathrm{rf}}\right)$. Our examples of PA design simulations need to be improved, but confirm our qualitative analysis and the potential benefits of EF-PER.

\section{Conclusion}

EF-PER, a new efficient linear transmitter architecture, has been presented, analyzed, and compared to the standard EER and ET architectures. It is based on a decomposition of the input signal in two components: a baseband modified envelope signal that controls the supply voltage of the RF PA and an RF bandpass signal that feeds the RF PA. The proposed EF-PER technique is especially suited to high PAPR signals such as OFDM signals.

As EER and ET, therefore, the EF-PER architecture is constituted of an envelope path and an RF path, but these paths have different characteristics for the three architectures.

To analyze and compare the architectures, we have conducted a numerical analysis with MATLAB and a PA electrical simulation under Agilent-ADS (with a transistor model). These analyses were done for high PAPR OFDMlike signals. In addition, the EF-PER signal statistics have been fully characterized analytically. A new global cost function $F$ has been proposed and presents an optimum value when setting the degree of freedom introduced by the EF-PER technique to $\alpha=0.5$.

In Sec. 2, the signals of the two paths have been characterized. It has been showed that, in comparison with ET and EER, EF-PER has the advantage of reducing the bandwidth, the PAPR/RCM and the normalized variance of the envelope signal. Compared to ET, the PAPR of the RF signal is decreased. Moreover, compared to EER, EF-PER is less sensitive to a time mismatch between the two paths, and the RF signal has a smaller bandwidth.

In Sec. 3, a realistic simulation of a $2 \mathrm{GHz}$ PA has been performed with Agilent-ADS, based on the non-linear model of an E-PHEMT (AVAGO). The qualitative analysis of the optimal operating point of the PA has been confirmed by simulations and the simulation confirmed the potential of EF-PER in terms of output power versus efficiency. EF-PER can also be considered as a potential solution for RF PA transistor size reduction (thus improving transistor frequency limit).

Section 2 and 3 provide two complementary points of view that justify the full relevance of EF-PER in practice. The interest of EF-PER has been showed to be the possibility of sharing out, or distributing, the critical parameters 
of the original input signal according to different criteria: Bandwidth, PAPR/RCM and normalized variance (NVAR). Future works will be to study the different parameters that can improve the method and to complete the EF-PER approach with PAPR reduction techniques and pre-distortion (DPD...).

\section{Appendix}

In this appendix, PDF, mean and variance are calculated for $Y$, the Rayleigh variable $X$ to the power $\alpha$.

$$
\begin{gathered}
X \propto \frac{x}{\sigma^{2}} e^{\left(-\frac{x^{2}}{2 \sigma^{2}}\right)} \ldots X, \text { Rayleigh } \quad Y=X^{\alpha} \\
\left.f_{X}(x) d x=f_{Y}(y) d y \quad d y x^{\alpha-1}\right) d x \\
f_{Y}(y)=f_{X}\left(x=y^{1 / \alpha}\right) \frac{1}{\left(\alpha y^{\alpha-1 / \alpha}\right)} \\
\text { with } Y=A(t)^{\alpha} \\
E(Y)=\int y f_{Y}(y) d y=m_{Y}(u)=\frac{1}{\alpha \cdot \sigma^{2}} u^{\frac{2-\alpha}{\alpha}} \exp \left(-\frac{u^{2 / \alpha}}{2 \sigma^{2}}\right) \\
=\int y \frac{1}{\alpha \cdot \sigma^{2}} y^{\frac{2-\alpha}{\alpha}} \exp \left(-\frac{y^{2 / \alpha}}{2 \sigma^{2}}\right) d y
\end{gathered}
$$

with $y=x^{\alpha}$ and $t=x^{2} / 2 . \sigma^{2}$

$$
\begin{gathered}
E(Y)=\left(2 \cdot \sigma^{2}\right)^{\alpha / 2} \int t^{\alpha / 2} e^{-t} d t=\left(2 \cdot \sigma^{2}\right)^{\alpha / 2} \cdot \Gamma\left(1+\frac{\alpha}{2}\right)=m_{x_{1}=x^{\alpha}} \\
E\left(Y-m_{Y}\right)^{2}=\int\left(y-m_{Y}\right)^{2} f_{Y}(y) d y=V A R_{Y=A^{\alpha}} \\
=\int\left(y-m_{Y}\right)^{2} \frac{1}{\alpha \cdot \sigma^{2}} y^{\frac{2-\alpha}{\alpha}} \exp \left(-\frac{y^{2 / \alpha}}{2 \sigma^{2}}\right) d y \\
=\int\left(x^{a}-m_{Y}\right)^{2} \frac{1}{\sigma^{2}} \exp \left(-\frac{x^{2}}{2 \sigma^{2}}\right) d x \\
E\left(Y-m_{Y}\right)^{2}=m_{Y}^{2}+\left(\alpha \cdot 2 \cdot \sigma^{2}\right) \cdot K \\
\left\{\begin{array}{l}
K=\left(2 \cdot \sigma^{2}\right)^{(\alpha-1)} \Gamma(\alpha)-\left(2 \cdot \sigma^{2}\right)^{\left(\frac{\alpha}{2}-1\right)} \Gamma\left(\frac{\alpha}{2}\right) m_{Y}
\end{array}\right.
\end{gathered}
$$

\section{References}

[1] JINGON JOUNG, CHIN KEONG HO, KOICHI ADACHI, SUMEI SUN. A survey on power-amplifier-centric techniques for spectrum- and energy-efficient wireless communications. IEEE Communication Surveys and Tutorials, 2015, vol. 17, no. 1, p. 315-333. DOI: 10.1109/COMST.2014.2350018

[2] DIET, A., VILLEGAS, M., BAUDOIN, G. EER-LINC RF transmitter architecture for high PAPR signals using SW power amplifiers. Physical Communication, Dec. 2008, vol. 1, no. 4, p. 248-254. DOI: 10.1016/j.phycom.2008.11.001

[3] GROE, J. A multimode cellular radio. IEEE Transactions on Circuits and Systems-II: Express Briefs, 2008, vol. 55, no. 3, p. 269-273. DOI: 10.1109/TCSII.2008.918997

[4] DIET, A., et al. RF transmitter architectures for nomadic multiradio: A review of the evolution towards fully digital solutions. Recent Patents on Electrical Engineering, 2013, vol. 6, no. 2, p. 79-94. DOI: 10.2174/22131116113069990006

[5] MORGAN, D. R., ZHENGXIANG MA, JAEHYEONG KIM, ZIERDT, M.G., PASTALAN, J. A generalized memory polynomial model for digital predistortion of RF power amplifiers. IEEE Transactions on Signal Processing, 2006, vol. 54, no. 10, p. 3852-3860. DOI: 10.1109/TSP.2006.879264

[6] WANG, F., OJO, A., KIMBALL, D., ASBECK, P., LARSON, L. Envelope tracking power amplifier with pre-distortion linearization for WLAN 802.11g. In IEEE MTT-S International Microwave Symposium Digest, 2004, vol. 3, p. 543-1546. DOI: 10.1109/MWSYM.2004.1338872

[7] RAAB, F.H. Efficiency of Doherty RF power-amplifier systems. IEEE Transactions on Broadcasting, 1987, vol. BC-33, no. 3, p. 77-83. DOI: 10.1109/TBC.1987.266625

[8] RAAB, F. H., et al. Power amplifiers and transmitters for RF and microwave. IEEE Transactions on Microwave Theory and Techniques, 2002, vol. 50, no. 3, p 814-826. DOI: $10.1109 / 22.989965$

[9] AMIRI, M. V., HELAOUI, M., GHANNOUCHI, F. M. On the estimation of power amplifier efficiency for modulated signals. In 2015 IEEE Topical Conference on Wireless and Radio Applications (PAWR). San Diego (CA, USA), Jan. 2015, p 1-4, DOI: 10.1109/PAWR.2015.7139203

[10] HANNIGTON, G., CHEN, P. F., ASBECK, P. M, LARSON, L. E. High-efficiency power amplifier using dynamic power-supply voltage for CDMA applications. IEEE Transactions on Microwave Theory and Techniques, 1999, vol. 47, no. 8, p. 1471-1476. DOI: $10.1109 / 22.780397$

[11] NIELSEN, M., LARSEN, T. Transmitter architecture based on $\Delta \Sigma$ modulation and SW power amplification. IEEE Transactions on Circuits and Systems II, 2007, vol. 54, no. 8, p. 735-739. DOI: 10.1109/TCSII.2007.899457

[12] CHOI, J., YIM, J., YANG, J., KIM, J., CHA, J., KANG, D., KIM, D., KIM, B. A $\triangle \Sigma$ digitized polar $\mathrm{RF}$ transmitter. IEEE Transactions on Microwave Theory and Techniques, 2007, vol. 55, no. 12 , p. 2679-2690. DOI: 10.1109/TMTT.2007.907137

[13] COX, D. C. Linear amplification with non-linear components, LINC method. IEEE Transactions on Communications, 1974, vol. COM-23, no. 10, p. 1942-1945. DOI: 10.5815/ijisa.2013.10.08

[14] COX, D. C. A high-efficiency RF transmitter using VCO-derived synthesis: CALLUM. In Proceedings of IEEE Radio and Wireless Conference RAWCON. Colorado Springs (CO,USA), 1998, p. 137-140. DOI: 10.1109/RAWCON.1998.709155

[15] JHENG, K.Y., CHEN, Y.J., WU, A.Y. Multilevel LINC system designs for power efficiency enhancement of transmitters. IEEE Journal of Selected Topics in Signal Processing, 2009, vol. 3, no. 3, p. 523-532. DOI: 10.1109/JSTSP.2009.2020949

[16] KAHN, R. Single sideband transmission by envelope elimination and restoration. Proceedings of the I.R.E., 1952, vol. 40, no. 7, p. 803-806. DOI: 10.1109/JRPROC.1952.273844

[17] NESIMOGLU, T., PARKER, S. C. J., MORRIS, K.A., MCGEEHAN, J. P. The performance and efficiency of envelope elimination and restoration transmitters for future multiple-input multiple-output wireless local area networks. IET Communica- 
tions, March 2008, vol. 2, no. 3, p. 473-483. DOI: 10.1049/ietcom:20070171

[18] RAAB, F. L-band transmitter using Kahn EER technique. IEEE Transactions on Microwave Theory and Techniques, 1998, vol. 46, no. 12 , p. 2220-2224. DOI: $10.1109 / 22.739200$

[19] OISHI, K. et al. A $1.95 \mathrm{GHz}$ fully integrated EER CMOS power amplifier with envelope/phase generator and timing aligner for WCDMA and LTE. In IEEE International Solid-State Circuits Conference Digest of Technical Papers (ISSCC). San Francisco (CA, USA), 2014, p. 60-61. DOI: 10.1109/ISSCC.2014.6757337

[20] BAUDOIN, G., BERLAND, C., VILlEGAS, M., DIET, A. Influence of time and processing mismatches between phase and envelope signals in linearization systems using EER. In IEEE MTT-S International Microwave Symposium Digest. Philadelphia (PA, USA), 2003, vol. 3, p. 2149-2152. DOI: 10.1109/MWSYM.2003.1210588

[21] JIN, S. et al. A highly efficient CMOS envelope tracking power amplifier using all bias node controls. IEEE Microwave and Wireless Components Letters, 2015, vol. 25, no. 8, p. 517-519. DOI: 10.1109/LMWC.2015.2440652

[22] Handset PA Linearization using Envelope Tracking. Nujira white paper. http://www.nujira.com.

[23] ANDERSON, D. R., CANTRELL, W. H. High-efficiency highlevel modulator for use in dynamic ET CDMA RF power amplifiers. In IEEE MTT-S International Microwave Symposium Digest. Phoenix (AZ, USA), 2001, vol. 3, p. 1509-1512. DOI: 10.1109/MWSYM.2001.967189

[24] CIDRONALI, A., MANES, G., GIOVANNELLI, N., VLASITS, T., HERNAMAN, R. Efficiency and linearity enhancements with envelope shaping control in dual-band envelope tracking GaAs PA. In Proceedings of the European Microwave Integrated Circuits Conference. Manchestr (UK), 2011, p. 308-311.

[25] HOVERSTEN, J., SCHAFER, S., ROBERG, M., NORRIS, M., MAKSIMOVIC, D., POPOVIC, Z. Codesign of PA, supply, and signal processing for linear supply-modulated RF transmitters. IEEE Transactions on Microwave Theory and Techniques, 2012, vol. 60, no. 6, p. 2010 2020. DOI: 10.1109/TMTT.2012.2187920

[26] LIE, D. Y. C., et al. Design of highly-efficient wideband RF polar transmitters using Envelope-Tracking (ET) for mobile WiMAX/Wibro applications. In Proceedings of the IEEE 8th International Conference on ASIC (ASICON '09). Changsha, (Hunan, China), Oct. 2009, p. 347-350. DOI: 10.1109/ASICON.2009.5351425

[27] GARCIA DOBLADO, J., et al. Cubic Metric Reduction for DCOOFDM visible light communication systems. Journal of Lightwave
Technology, 2015, vol. 33, no. 10, p. 1971-1978. DOI: 10.1109/JLT.2015.2402755

\section{About the Authors ...}

Antoine DIET was born in France in 1979. He graduated from ESIEE Paris in 2001 (Eng.) and received the Ph.D. degree from the University of Paris-Est in 2005 (Dr.) in the domain of Radio-Electronics Systems. He is currently associate professor in the campus of Paris Saclay and performs his research in the GeePS research laboratory (UMR 8507, CNRS, CentraleSupélec, Univ. Paris Sud, UPMC). His research topics include antennas, UWB, RFID/NFC and wireless architectures.

Geneviève BAUDOIN was born in France in 1954. She graduated from the École Nationale Supérieure des Télécommunications (ENST), Paris, France, in 1977 and received the Habilitation for PHD direction from the University of Marne La Vallée in 2000. She was lecturer at the university of Paris-Ouest; then she joined the Philips Research laboratory in France. Since 1980, she has been with ESIEE Paris. She is presently Professor with the Department of System Engineering and chair of the department. She is a member of the ESYCOM research laboratory (EA 2552) of Université Paris-Est. Her research and teaching interests include wireless communications/transceivers and digital signal processing.

Samson LASAULCE (Prof.) received his BSc and Agrégation degree in Physics from École Normale Supérieure (Cachan) and his MSc and Ph.D. in Signal Processing from Telecom Paris. He has been working with Motorola Labs (1999-2001) and with Orange Labs (2002-2003). Since 2004, he has joined the CNRS and Supélec as a Senior Researcher. Since 2004, he has also been a professor with École Polytechnique. Samson Lasaulce is the recipient of several paper awards. He is an author of the book "Game Theory and Learning for Wireless Networks: Fundamentals and Applications". 